DFTT 20/98

NORDITA 98/40-HE

KUL-TF-98/25

May 1998

\title{
The Lorentz force between D0 and D6 branes in string and $M($ atrix $)$ theory
}

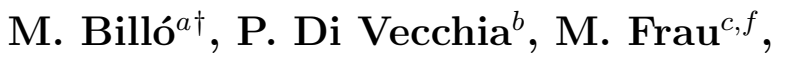 \\ A. $\operatorname{Lerda}^{d, c, f}, \mathbf{R}$. Russo ${ }^{e, f}$ and S. Sciuto ${ }^{c, f}$ \\ a Instituut voor theoretische fysica, \\ Katholieke Universiteit Leuven, B-3001 Leuven, Belgium \\ ${ }^{b}$ NORDITA, Blegdamsvej 17, DK-2100 Copenhagen Ø, Denmark \\ ${ }^{c}$ Dipartimento di Fisica Teorica, Università di Torino \\ ${ }^{d}$ Dipartimento di Scienze e Tecnologie Avanzate \\ Università di Torino, sede di Alessandria \\ e Dipartimento di Fisica, Politecnico di Torino \\ f I.N.F.N., Sezione di Torino, Via P. Giuria 1, I-10125 Torino, Italy
}

\begin{abstract}
We use different techniques to analyze the system formed by a D0 brane and a D6 brane (with background gauge fields) in relative motion. In particular, using the closed string formalism of boosted boundary states, we show the presence of a term linear in the velocity, corresponding to the Lorentz force experienced by the D0 brane moving in the magnetic background produced by the D6 brane. This term, that was missed in previous analyses of this system, comes entirely from the R-R odd spin structure and is also reproduced by a $\mathrm{M}($ atrix $)$ theory calculation.
\end{abstract}

*Work partially supported by the European Commission TMR programme ERBFMRX-CT960045 in which R.R. is associated to Torino University, and by MURST.

${ }^{\dagger}$ e-mail: billo@tfdec1.fys.kuleuven.ac.be 


\section{Introduction}

The interaction between two D-branes [1] can be computed in many different ways: from the string point of view, the short-distance physics is entirely described by the open strings stretched between the branes [2], while, from the M(atrix) theory point of view, it is reproduced by super Yang-Mills calculations in $0+1$ dimensions [3] 1. On the other hand, the long-distance behavior of D-branes is conveniently captured by the closed string boundary state formalism 2], where the D-branes are described by BRST invariant states and the interaction is computed by connecting them with closed string propagators [6, 7, 8, 5].

In this paper we use different techniques to analyze the system formed by a static D6 brane and a D0 brane moving along a direction transverse to the D6 brane [9]. In all cases, we find that the interaction contains a linear term in the velocity that was missed in previous analyses from both the string theory and the $\mathrm{M}$ (atrix) theory side [10, 11, 12, 13. . However, this term has a very simple classical interpretation: in fact, since a D0 and a D6 brane are Hodge dual to each other, they are the analogue in ten dimensions of pointlike electric and magnetic charges in four dimensions; thus a velocity dependent interaction is expected, since it represents the Lorentz force experienced by a particle (the D0 brane) moving in a magnetic background (produced by the D6 brane). The presence of this term, in a similar physical situation, has been recently pointed out in Ref. [14, where the authors studied the off-diagonal interaction between a D3 brane and a D3 brane-antibrane system using string techniques. In particular, they have related the magnetic force to the presence of the odd spin structure of the R-R sector that usually gives no contribution.

This remark suggests that there should be a relation between a system of a D0 and a D6 brane in relative motion, and the one formed by a D0 and a D8 brane at rest. In fact also in the latter case the odd spin structure gives a non vanishing contribution, as it happens, in general, for the interaction between two parallel D-branes when the difference of their dimensionalities $\nu$ is 8 . Since these systems satisfy the BPS no-force condition [1], a non vanishing R-R contribution is needed to compensate the gravitational interactions of the NS-NS sector. On the other hand, a R-R interaction between two D-branes of different dimensionality is difficult to understand because the two D-branes seem to couple to different R-R potentials. However, as explained in Ref. [5], the R-R interaction in the $\nu=8$ brane systems arises entirely from the odd spin structure where the presence of a nontrivial regulator on the matter and superghost zero-modes leads to duality relations between different R-R potentials. As we shall see, a similar mechanism can explain the non-vanishing R-R interaction between a D6 brane and a moving D0 brane. On

\footnotetext{
${ }^{1}$ For a review of the role of gauge theories in the D-brane physics see Ref. 四 and references therein.

${ }^{2} \mathrm{~A}$ detailed discussion of the boundary state formalism can be found in Ref. [5] and references therein.
} 
the other hand, from the M(atrix) theory point of view, the term corresponding to the odd spin structure in string computation is due to the presence of an unpaired eigenvector of the fermionic lagrangian [15, 16] which is a feature common to both the D0-D6 and D0-D8 brane systems.

In order to make the tight connection between the two systems immediately evident, let us start by describing them at low energies in the source-probe framework [17]. The key ingredient in this approach is that different $\mathrm{R}-\mathrm{R}$ form potentials may describe the same physical field and should be identified. This can be seen by studying in detail the massless R-R sector in the asymmetric picture as was recently done in Ref. [5], where it was shown that the Hilbert space in this sector contains two dimensional subspaces with degenerate metric: removing the null-states, one is led to identify pairs of different vectors. Normally this identification reduces to the usual Hodge duality for the R-R potentials, but the string analysis reveals that, for off-shell states, there are also non-trivial relations. In particular, the $\mathrm{R}-\mathrm{R}$ potentials of a one-form coupled to a D0 brane and of a nine-form coupled to a D8 brane with non-vanishing momentum in the 9 th direction are identified:

$$
A_{0}=-A_{012 \ldots 8}
$$

Using this result, the presence of a R-R interaction in the D0-D8 system is interpreted as due to the fact that the $\mathrm{R}-\mathrm{R}$ charges of the two branes are identified by a duality relation, and thus produce the same R-R potential. According to this analysis, the R-R interaction of the D0-D8 system has then a simple microscopic description as the usual Coulomb-like force between D-branes. As a check on the validity of this interpretation, we now show that Eq. (1.1) implies that the R-R interaction in the D0-D8 brane system leads to the static potential

$$
V_{08}(r)=\frac{1}{4 \pi \alpha^{\prime}} r
$$

where $r$ is the distance between the two branes, whose presence explains the by-now well known phenomenon of the creation of a fundamental string when the D0 brane passes through the D8 brane [19]. Let us consider the low-energy effective action of a D0 brane with tension $\tau_{0}$ and charge $\mu_{0}$ in presence of a R-R background, which in the static gauge reads

$$
S_{e f f}=-\tau_{0} \int d \tau \sqrt{1-\left(\dot{x}^{i}\right)^{2}}-\mu_{0} \int d \tau\left(A_{0}+\dot{x}^{i} A_{i}\right)
$$

Then, if we use the identification (1.1), we can view the D8 brane as a source for the potential experienced by the D0 brane, so that

$$
A_{0}=-A_{012 \ldots 8}=\frac{\mu_{8}}{2} r
$$

where $\mu_{p}=\sqrt{2 \pi}\left(2 \pi \alpha^{\prime}\right)^{3-p}$ is the coupling of a $(p+1)$-form R-R field to a $\mathrm{D} p$ brane. Inserting the potential (1.4) into the Wess-Zumino part of the Born-Infeld action 
(1.3) and using the identity $\mu_{0} \mu_{8}=\frac{1}{2 \pi \alpha^{\prime}}$, we see that the term proportional to $A_{0}$ immediately reproduces Eq. (1.2). This simple argument provides a nice consistency check of our identification of the potentials produced by a zero and an eight brane to explain the appearance of the term in Eq. (1.2).

In order to apply a similar procedure to the D0-D6 brane system, one has to find whether there exists some relation between the R-R potentials generated by a D6 brane and those coupled to a moving D0 brane. For the sake of simplicity, we suppose that the world volume of the D6 brane lies in the directions (0)123456, and that the D0 brane velocity $v$ is in the 9 th direction with an impact parameter $\vec{b}$ in the (78) plane. In this configuration, the standard Hodge duality relations read $\partial_{7} A_{012 \ldots 6}=\partial_{8} A_{9}$ and $\partial_{8} A_{012 \ldots 6}=-\partial_{7} A_{9}$, where $A_{012 \ldots 6}$ is the seven-form emitted by the D6 brane and $A_{9}$ is the component of the one-form coupled to the moving D0 brane. Then, using the explicit expression of $A_{012 \ldots 6}$, we can write

$$
A_{9}(\vec{b}, v \tau)=-\int^{\vec{b}}\left(d x^{7} \partial_{8}-d x^{8} \partial_{7}\right)\left(\frac{\mu_{6}}{4 \pi} \frac{1}{r}\right),
$$

where we have defined $r^{2}=\vec{x} \cdot \vec{x}+v^{2} \tau^{2}$. Inserting this relation inside the D0 brane effective action and recalling that $\mu_{0} \mu_{6}=2 \pi$, the last term of Eq. (1.3) becomes

$$
-\int^{\vec{b}}\left(d x^{7} \partial_{8}-d x^{8} \partial_{7}\right)\left(\int_{-\infty}^{\infty} d \tau V_{06}(r)\right)
$$

where we have defined the velocity-dependent long-range "magnetic" potential

$$
V_{06}(r)=-\frac{v}{2 r}
$$

which, through Eq. (1.6), describes a Lorentz-like interaction.

In Section 2, using boundary states and following the procedure discussed in detail in Ref. [5], we compute the interaction between a D6 brane (with a background magnetic field) at rest and a moving D0 brane. In particular we focus on the R$\mathrm{R}$ sector where we expect to find from the odd spin structure a non vanishing contribution of the type (1.7). In Section 3, we consider the same system from the $\mathrm{M}$ (atrix) theory point of view and show that the same term coming from the odd spin structure in string computation is obtained from a contribution of the fermionic determinants. At first sight, this agreement between the two different approaches may appear surprising: in fact, the M(atrix) theory results usually agree with those obtained by supergravity calculations only in the infinite momentum frame and thus it seems that there is no reason to explain why, for the odd spin structure contribution, the long distance result coming from the boundary state should always coincide with the short distance M(atrix) calculation. However, in Section 2, performing the calculation of the R-R sector, we will find that in the oddspin structure, all the stringy massive states give no contribution; this property is not peculiar of the D0-D6 brane system, but is a general feature common to all the 
interactions between two arbitrary boundary states. The fact that the whole result comes from the zero-modes of the various fields ensures that the behavior is the same at all scales. In fact, in order to deduce the short distance behavior from the results written in the closed string channel, one should usually make the modular transformation $t \rightarrow 1 / t$; however, when the massive modes give no contribution, this transformation simply maps the contribution of the massless string states into that of the massless open string and thus for the odd-spin structure the "duality" between loops and tree diagrams holds also at the field theory level.

\section{String theory calculation}

In the boundary state formalism, a $\mathrm{D} p$ brane is described by a BRST invariant state $|B\rangle$, which inserts a boundary on the string world-sheet and represents the source for the closed strings emitted by the brane. As discussed in Ref. [5], the boundary state both in the NS-NS and in the R-R sector of the fermionic string can be written as the product of a matter part and a ghost part

$$
|B, \eta\rangle=\frac{T_{p}}{2}\left|B_{\mathrm{mat}}, \eta\right\rangle\left|B_{\mathrm{g}}, \eta\right\rangle
$$

where $\eta= \pm 1, T_{p}=\sqrt{\pi}\left(2 \pi \sqrt{\alpha^{\prime}}\right)^{3-p}$ is the brane tension, and

$$
\left|B_{\mathrm{mat}}, \eta\right\rangle=\left|B_{X}\right\rangle\left|B_{\psi}, \eta\right\rangle \quad, \quad\left|B_{\mathrm{g}}, \eta\right\rangle=\left|B_{\mathrm{gh}}\right\rangle\left|B_{\mathrm{sgh}}, \eta\right\rangle
$$

The explicit expressions of the various components of the boundary state are given in Ref. [5]. Here, we simply recall the general structure of the matter sector ?, namely

$$
\left|B_{X}\right\rangle=\exp \left[-\sum_{n=1}^{\infty} \frac{1}{n} \alpha_{-n} \cdot S \cdot \tilde{\alpha}_{-n}\right]\left|B_{X}\right\rangle^{(0)}
$$

for the bosonic part, and

$$
\begin{gathered}
\left|B_{\psi}, \eta\right\rangle_{\mathrm{NS}}=\exp \left[\mathrm{i} \eta \sum_{m=1 / 2}^{\infty} \psi_{-m} \cdot S \cdot \tilde{\psi}_{-m}\right]|0\rangle, \\
\left|B_{\psi}, \eta\right\rangle_{\mathrm{R}}=\exp \left[\mathrm{i} \eta \sum_{m=1}^{\infty} \psi_{-m} \cdot S \cdot \tilde{\psi}_{-m}\right]\left|B_{\psi}, \eta\right\rangle_{\mathrm{R}}^{(0)},
\end{gathered}
$$

respectively for the NS-NS and R-R sectors of the fermionic part. In these expressions, the matrix $S_{\mu \nu}$ encodes the boundary conditions characterizing the $\mathrm{D} p$ brane which depend on its velocity and the presence of gauge fields on its world-volume. The superscript ${ }^{(0)}$ in Eqs. (2.3) and (2.5) denotes the zero-mode contributions which also depend on the boundary conditions. Let us now give some details for

\footnotetext{
${ }^{3}$ The ghost contribution is the same as in Ref. [5], and we do not rewrite it here again.
} 
the configuration we want to study. The matrix $S$ for a D0 brane moving with a velocity $v$ along the 9 -th direction, is given by the following block-diagonal form

$$
S=\operatorname{diag}\left(-\mathcal{V}_{09},-\mathbb{1}_{12},-\mathbb{1}_{34},-\mathbb{1}_{56},-\mathbb{1}_{78}\right)
$$

where $\mathbb{1}_{i j}$ is the identity in the $i$-th and $j$-th directions, and $\mathcal{V}_{09}$ is a $2 \times 2$ matrix, acting in the 0 -th and 9 -th directions, given by

$$
\mathcal{V}_{09}=\left(\begin{array}{cc}
\frac{1+v^{2}}{1-v^{2}} & \frac{2 v}{1-v^{2}} \\
\frac{2 v}{1-v^{2}} & \frac{1+v^{2}}{1-v^{2}}
\end{array}\right)=\left(\begin{array}{cc}
\cosh (2 \pi \nu) & \sinh (2 \pi \nu) \\
\sinh (2 \pi \nu) & \cosh (2 \pi \nu)
\end{array}\right)
$$

where

$$
v=\tanh (\pi \nu)
$$

This result can be derived by applying the boost operator $\exp \left(\mathrm{i} \pi \nu J^{09}\right)\left(J^{\rho \sigma}\right.$ being the generators of the Lorentz transformations) to the boundary state of a D0 brane at rest, as explicitly done in Ref. [6]. The action of the boost operator on the zero-mode part of the boundary state yields for the bosonic sector

$$
\left|B_{X}\right\rangle^{(0)}=\sqrt{1-v^{2}} \delta^{(8)}(\hat{q}-y) \delta\left(\hat{q}^{0} v+\hat{q}^{9}\right)|k=0\rangle
$$

where $\delta^{(8)}$ denotes the $\delta$-function that fixes the position of the D0 brane in all transverse directions except the 9-th along which the motion takes place. In the fermionic R-R sector, instead, one finds

$$
\left|B_{\psi}, \eta\right\rangle_{\mathrm{R}}^{(0)}=\mathcal{M}_{A B}^{(\eta)}|A\rangle|\tilde{B}\rangle
$$

where

$$
\mathcal{M}^{(\eta)}=C \Gamma^{0}\left(\frac{1-v \Gamma^{09}}{\sqrt{1-v^{2}}}\right)\left(\frac{1+\mathrm{i} \eta \Gamma_{11}}{1+\mathrm{i} \eta}\right),
$$

with $C$ being the charge conjugation matrix.

Let us now turn to the D6 brane. To be able to compare the string results with the $\mathrm{M}$ (atrix) theory calculation performed in the next section, we switch on a constant magnetic field on the world volume of a static D6 brane. In this way, we actually describe a $(6+4+2+0)$-bound state. The matrix $S$ corresponding to this configuration is given by the following block-diagonal form

$$
S=\operatorname{diag}\left(-\mathbb{1}_{09}, \mathcal{F}_{12}, \mathcal{F}_{34}, \mathcal{F}_{56},-\mathbb{1}_{78}\right),
$$

where the $2 \times 2$ matrix $\mathcal{F}_{i j}$, acting in the $i$-th and $j$-th directions, is 1

$$
\mathcal{F}_{i j}=\left(\begin{array}{cc}
\frac{1-f^{2}}{1+f^{2}} & \frac{2 f}{1+f^{2}} \\
-\frac{2 f}{1+f^{2}} & \frac{1-f^{2}}{1+f^{2}}
\end{array}\right)=\left(\begin{array}{cc}
\cos (2 \pi \epsilon) & \sin (2 \pi \epsilon) \\
-\sin (2 \pi \epsilon) & \cos (2 \pi \epsilon)
\end{array}\right)
$$

\footnotetext{
${ }^{4}$ For simplicity we have taken the same value of $f$ in all three blocks. The generalization to different values is straightforward.
} 
where

$$
f=\tan (\pi \epsilon)
$$

This result can be obtained by generalizing the construction described in Ref. [8], where it was shown how to create a bound state of D-branes by performing a rotation followed by a T-duality transformation. Correspondingly, the zero-mode piece of the bosonic boundary state is found to be

$$
\left|B_{X}\right\rangle^{(0)}=\left(\sqrt{1+f^{2}}\right)^{3} \delta^{(3)}(\hat{q}-y)|k=0\rangle
$$

where the delta function fixes the position of the D6 brane in its three transverse directions (i.e. 7,8,9), whereas for the R-R sector it is like in Eq. (2.10) with the matrix $\mathcal{M}^{(\eta)}$ given by

$$
\mathcal{M}^{(\eta)}=C \Gamma^{01 \ldots 56}\left[\left(\frac{1-f \Gamma^{12}}{\sqrt{1+f^{2}}}\right)\left(\frac{1-f \Gamma^{34}}{\sqrt{1+f^{2}}}\right)\left(\frac{1-f \Gamma^{56}}{\sqrt{1+f^{2}}}\right)\right]\left(\frac{1+\mathrm{i} \eta \Gamma_{11}}{1+\mathrm{i} \eta}\right) .
$$

This matrix clearly exhibits the structure of a bound state of D6, D4, D2 and D0 branes, corresponding to the terms with zero, two, four and six $\Gamma$ matrices in the square brackets, and is formally similar to the boosted matrix in Eq. (2.11).

We are now in the position of computing the scattering amplitude

$$
\mathcal{A}=\left\langle B_{1}|D| B_{2}\right\rangle
$$

where $D$ is the closed string propagator, and $\left|B_{1}\right\rangle$ and $\left|B_{2}\right\rangle$ are the GSO projected boundary states describing the moving D0 brane and the D6 brane bound state respectively. Let us begin by discussing the NS-NS contribution to $\mathcal{A}$. Proceeding as explained in detail in Refs. [5, 6] we get

$$
\begin{aligned}
\mathcal{A}_{\mathrm{NS}-\mathrm{NS}}= & \frac{\sqrt{1-v^{2}}}{32 \sqrt{2 \pi \alpha^{\prime}}} \int_{-\infty}^{\infty} d \tau \int_{0}^{\infty} \frac{d t}{t^{3 / 2}} e^{t} \exp \left[-\frac{(\Delta y)^{2}+v^{2} \tau^{2}}{2 \alpha^{\prime} t}\right] \\
\times & \left\{\prod_{n=1}^{\infty} \frac{\operatorname{det}\left(1+q^{2 n-1} S_{2}\left(S_{1}\right)^{T}\right)}{\operatorname{det}\left(1-q^{2 n} S_{2}\left(S_{1}\right)^{T}\right)} \frac{\left(1-q^{2 n}\right)^{2}}{\left(1+q^{2 n-1}\right)^{2}}\right. \\
& \left.-\prod_{n=1}^{\infty} \frac{\operatorname{det}\left(1-q^{2 n-1} S_{2}\left(S_{1}\right)^{T}\right)}{\operatorname{det}\left(1-q^{2 n} S_{2}\left(S_{1}\right)^{T}\right)} \frac{\left(1-q^{2 n}\right)^{2}}{\left(1-q^{2 n-1}\right)^{2}}\right\},
\end{aligned}
$$

where $q=\mathrm{e}^{-t}$, and $S_{1}$ and $S_{2}$ are given respectively by Eqs. (2.6) and (2.12). The last two lines of Eq. (2.18) represent the contribution of the non-zero modes of the NS-NS closed string states exchanged between the two D-branes. The two terms in braces correspond to the two NS-NS spin structures and the relative minus sign assures the cancellation of the tachyon. We remark that to compute this part, it is not necessary to specify the detailed form of the matrices $S$, thus the non-zero mode contribution always takes this form for any brane configuration. Upon inserting the 
explicit expressions for $S_{1}$ and $S_{2}$, and rescaling the modular parameter $t \rightarrow \pi t$, one can rewrite Eq. (2.18) in terms of Jacobi $\theta$ functions and get

$$
\begin{aligned}
\mathcal{A}_{N S-N S} & =\frac{v}{2 \pi \sqrt{2 \alpha^{\prime}}} \int_{-\infty}^{\infty} d \tau \int_{0}^{\infty} \frac{d t}{t^{3 / 2}} \exp \left[-\frac{r^{2}}{2 \pi \alpha^{\prime} t}\right] \\
& \times\left\{\mathrm{i} \frac{\theta_{3}(\mathrm{i} \nu \mid \mathrm{i} t)}{\theta_{1}(\mathrm{i} \nu \mid \mathrm{i} t)}\left(\frac{\theta_{4}(\epsilon \mid \mathrm{i} t)}{\theta_{2}(\epsilon \mid \mathrm{i} t)}\right)^{3}-\mathrm{i} \frac{\theta_{4}(\mathrm{i} \nu \mid \mathrm{i} t)}{\theta_{1}(\mathrm{i} \nu \mid \mathrm{i} t)}\left(\frac{\theta_{3}(\epsilon \mid \mathrm{i} t)}{\theta_{2}(\epsilon \mid \mathrm{i} t)}\right)^{3}\right\},
\end{aligned}
$$

where $r^{2}=(\Delta y)^{2}+v^{2} \tau^{2}$, and the arguments of the $\theta$ functions are given by Eqs. (2.8) and (2.14).

Let us now turn to the R-R sector. In this case it is easier to perform the calculation before the GSO projection. After some straightforward algebra we find

$$
\begin{aligned}
\mathrm{R}\left\langle B^{1}, \eta_{1}|D| B^{2}, \eta_{2}\right\rangle_{\mathrm{R}} & =\frac{1}{16 \sqrt{2 \pi \alpha^{\prime}}} \int_{-\infty}^{\infty} d \tau \int_{0}^{\infty} \frac{d t}{t^{3 / 2}} \exp \left[-\frac{r^{2}}{2 \alpha^{\prime} t}\right] \\
& \times \sqrt{1-v^{2}}\left(\sqrt{1+f^{2}}\right)^{3} \stackrel{(0)}{\mathrm{R}}\left\langle B^{1}, \eta_{1} \mid B^{2}, \eta_{2}\right\rangle_{\mathrm{R}}^{(0)} \\
& \times\left\{\delta_{\eta_{1} \eta_{2}, 1}+\delta_{\eta_{1} \eta_{2},-1} \prod_{n=1}^{\infty} \frac{\operatorname{det}\left(1+q^{2 n} S_{2}\left(S_{1}\right)^{T}\right)}{\operatorname{det}\left(1-q^{2 n} S_{2}\left(S_{1}\right)^{T}\right)} \frac{\left(1-q^{2 n}\right)^{2}}{\left(1+q^{2 n}\right)^{2}}\right\} .
\end{aligned}
$$

In this expression ${ }_{\mathrm{R}}^{(0)}\left\langle B^{1}, \eta_{1} \mid B^{2}, \eta_{2}\right\rangle_{\mathrm{R}}^{(0)}$ denotes the zero-mode contribution, while the terms in braces are due to the non-zero modes of the $\mathrm{R}-\mathrm{R}$ states exchanged between the two D-branes. Notice that in the piece proportional to $\delta_{\eta_{1} \eta_{2}, 1}$ there is an exact cancellation between the bosonic and fermionic non-zero modes while they survive in the piece proportional to $\delta_{\eta_{1} \eta_{2},-1}$. This cancellation is an algebraic fact due to world-sheet supersymmetry, and always occurs for any D-brane configuration, i.e. independently of the explicit expressions of the matrices $S_{1}$ and $S_{2}$. Since, as one can see, the term proportional to $\delta_{\eta_{1} \eta_{2}, 1}$ corresponds to the odd R-R spin structure, while the term proportional to $\delta_{\eta_{1} \eta_{2},-1}$ corresponds to the even R-R spin structure, we expect a very different behavior of the respective interactions. We will return to this crucial point later.

To evaluate the zero mode contribution in Eq. (2.20) one has to follow the procedure described in detail in Ref. [5], which in particular requires not to separate the fermionic and superghost zero-modes and to use a regulator inside the inner product f. Following this procedure, it is easy to find that

$$
{ }_{\mathrm{R}}^{(0)}\left\langle B_{\psi}^{1}, \eta_{1} \mid B_{\psi}^{2}, \eta_{2}\right\rangle_{\mathrm{R}}^{(0)}=-16 \frac{1}{\sqrt{1-v^{2}}}\left(\frac{1}{\sqrt{1+f^{2}}}\right)^{3}\left(f^{3} \delta_{\eta_{1} \eta_{2},-1}-v \delta_{\eta_{1} \eta_{2}, 1}\right) .
$$

\footnotetext{
${ }^{5}$ The fermionic regulator that must be used in this case is $x^{\Gamma^{09}} x^{\Gamma^{12}} x^{\Gamma^{34}} x^{\Gamma^{56}} x^{\Gamma^{78}}$. This expression, which slightly differs from the one used in Ref. [5], namely $x^{\Gamma^{09}} x^{-\mathrm{i} \Gamma^{12}} x^{-\mathrm{i} \Gamma^{34}} x^{-\mathrm{i} \Gamma^{56}} x^{-\mathrm{i} \Gamma^{78}}$, yields a well-defined and unitary inner-product also in those cases, like the present one, in which the ghosts and superghosts effectively remove two spatial directions (e.g. the 7 th and 8th).
} 
Inserting this result in Eq. (2.20), using the explicit expressions for the matrices $S_{1}$ and $S_{2}$, performing the GSO projection and introducing the Jacobi $\theta$ functions, we obtain

$$
\begin{aligned}
\mathcal{A}_{\mathrm{R}-\mathrm{R}} & =\frac{v}{2 \pi \sqrt{2 \alpha^{\prime}}} \int_{-\infty}^{\infty} d \tau \int_{0}^{\infty} \frac{d t}{t^{3 / 2}} \exp \left[-\frac{r^{2}}{2 \pi \alpha^{\prime} t}\right] \\
& \times\left\{-\mathrm{i} \frac{\theta_{2}(\mathrm{i} \nu \mid \mathrm{i} t)}{\theta_{1}(\mathrm{i} \nu \mid \mathrm{i} t)}\left(\frac{\theta_{1}(\epsilon \mid \mathrm{i} t)}{\theta_{2}(\epsilon \mid \mathrm{i} t)}\right)^{3}+1\right\} .
\end{aligned}
$$

Performing the modular transformation $t \rightarrow 1 / t$ one can check that our results (2.19) and (2.22) agree with the phase shift calculated in Ref. [10, 11] using the open string formalism, except for the odd spin structure contribution which was missed in the previous calculations. It is interesting to remark that the same expression (2.22) can be obtained using the light-cone methods described in Ref. [18], thus providing a strong consistency check on our results. On the other hand, our analysis clearly shows, the configuration of a D0 brane scattering off a D6 brane has the same R-R zero-mode content as the D0-D8 brane system, where it is known that the odd spin structure is non vanishing [19, 5]. On the other hand, the presence of a contribution from the odd spin structure and its physical relevance have been recently discussed in Ref. [14 for a system similar to the one we are considering.

From the total amplitude $\mathcal{A}_{\mathrm{NS}-\mathrm{NS}}+\mathcal{A}_{\mathrm{R}-\mathrm{R}}$, we can now define the long range potential between the two D-branes due to the exchange of the massless closed string states. To do so, we treat separately the even and odd spin structures, since they correspond to different types of interactions. Indeed, the even spin structures describe the usual interplay between the gravitational attraction from the NS-NS sector and the Coulomb-like electric repulsion from the $\mathrm{R}-\mathrm{R}$ sector, while the odd spin structure accounts for the Lorentz-like electro-magnetic interaction. Expanding Eqs. (2.19) and (2.22) for $t \rightarrow \infty$ in order to select the contribution of the massless closed string states, we can introduce an even and odd long range potential given respectively by

$$
V_{\text {even }}(r)=-\frac{v}{8}\left(\frac{\cosh 2 \pi \nu-3 \cos 2 \pi \epsilon-4 \cosh \pi \nu \sin ^{3} \pi \epsilon}{\sinh \pi \nu \cos ^{3} \pi \epsilon}\right) \frac{1}{r}
$$

and

$$
V_{\text {odd }}(r)=-\frac{v}{2 r} \text {. }
$$

The potential $V_{\text {even }}$ agrees with the one computed in Ref. [11], whereas the potential $V_{\text {odd }}$ coincides with the one in Eq. (1.7) obtained with the probe-source approach. Besides a different physical interpretation, the two potentials (2.23) and (2.24) have also a very different behavior. Indeed, $V_{\text {odd }}$ has the same $r$ dependence at all scales, because it arises from the odd R-R spin structure in which the non-zero modes exactly cancel. This peculiar feature guarantees that the potential at large distances, where only the lightest closed string modes contribute, is exactly the 
same as the potential at small distances where only the lightest open string modes contribute. For this reason, one should expect that the Lorentz-like potential (2.24) be reproduced also from a $\mathrm{M}$ (atrix) theory calculation. On the other hand, the potential $V_{\text {even }}$ arises from the even spin structures in which the non-zero modes do not cancel. Thus, the long distance potential (2.23) is different from the short distance one obtained by truncating the open string to its massless level. However, there is a regime in which the non-zero modes can be neglected also for the even spin structures so that an agreement between the long and short-distance behaviors can be obtained, namely for small velocities and high fields. To discuss this regime, it is convenient to set $\epsilon=1 / 2-c / \pi$ and expand the phase shift of the even spin structures for $\nu, c \rightarrow 0$. Then, one can explicitly check from Eqs. (2.19) and (2.22) that only the massless closed string states contribute to the leading order in $\nu$ and $c$, whereas the massive modes give contributions of higher order. Thus, only the leading order term in the expansion of Eq. (2.23), namely

$$
V_{\mathrm{even}}(r) \simeq-\frac{v^{4}+6 v^{2} c^{2}-3 c^{4}}{16 c^{3}} \frac{1}{r}
$$

where $c \simeq 1 / f$, represents a potential valid at all distances. Indeed, the same expression arises from a $\mathrm{M}$ (atrix) theory calculation [10, 11, 12, 13] in the infinite momentum frame. In the following section, we show that the agreement between the closed string calculation and the M(atrix) theory actually occurs, as it should, also for the Lorentz-like potential (2.24) without making any approximation or choosing the infinite momentum frame.

\section{$3 \mathrm{M}($ atrix $)$ theory calculation}

As usual, the starting point for $\mathrm{M}$ (atrix) theory calculations is the 10D lagrangian of super Yang-Mills reduced to 1+0 dimensions; however, in this section, we focus only on the fermionic part of this lagrangian because our purpose is to find the term corresponding to the odd spin structure which can only come from the fermionic sector. On the other hand, the bosonic sector of this M(atrix) theory has been analyzed and discussed in Ref. [10, 11]. Thus we just consider the following lagrangian 4

$$
L_{f}=\frac{1}{2} \operatorname{Tr}\left(\bar{\psi} \mathrm{i} \Gamma^{0} \partial_{t} \psi-\bar{\psi} \Gamma^{i}\left[X_{i}, \psi\right]\right),
$$

where $X^{i}$ are the bosonic degrees of freedom, while $\psi$ are 10-dimensional MajoranaWeyl spinors. In the case of a two D-brane system, these fields are $U(2)$ matrices with well defined classical values. We can describe a system of a D0 and a D6 brane with relative velocity $v$ in the 9 th direction and impact parameter $b$ in the 8th direction, by using the following background matrices for the bosonic fields

$$
X_{2 i-1}=\left(\begin{array}{cc}
Q_{i} & 0 \\
0 & 0
\end{array}\right), \quad X_{2 i}=\left(\begin{array}{cc}
P_{i} & 0 \\
0 & 0
\end{array}\right)
$$




$$
X_{7}=0, \quad X_{8}=\left(\begin{array}{cc}
b & 0 \\
0 & 0
\end{array}\right), \quad X_{9}=\left(\begin{array}{cc}
0 & 0 \\
0 & v t
\end{array}\right),
$$

where $i=1,2,3$, and

$$
\left[Q_{i}, P_{j}\right]=\mathrm{i} c_{i} \delta_{i j}
$$

and trivial classical values for the fermionic variables. Note that the above choice for the background fields does not describe a pure D-brane, but a D6 brane with a constant magnetic field switched on, that is a $(6+4+2+0)$ bound state, just like the one discussed in the previous section. Thus, besides the magnetic contribution, that is linear in the velocity, the $\mathrm{M}$ (atrix) theory result will also contain an electric term due to the interaction between the moving D0 brane and the D0 branes contained in the bound state. However, it is easy to separate the two contributions since the magnetic force is independent of $c_{i}$, that are related to the gauge fields switched on the D6 brane.

Now we choose an explicit representation for $\Gamma^{\mu}$, by taking $\Gamma^{0}=\mathrm{i} \sigma_{1} \otimes \gamma^{8}$, $\Gamma^{i}=\sigma_{1} \otimes \gamma^{i}(i=1, \ldots, 6), \Gamma^{7}=\sigma_{2} \otimes 11, \Gamma^{8}=\sigma_{1} \otimes \gamma^{9}, \Gamma^{9}=\sigma_{1} \otimes \gamma^{7}$, where $\gamma^{i}$ are the eight $16 \times 16$ gamma matrices of $S O(8)$ and $\gamma^{9}=\gamma^{1} \cdots \gamma^{8}$. The reason for this unusual choice is simply a matter of later convenience, since with this representation the D0-D6 problem becomes formally similar to the D0-D8 problem studied in Ref. [15. Indeed, using Eq. (3.2), the lagrangian (3.1) can be rewritten as follows

$$
\begin{aligned}
L_{f}= & -\frac{\mathrm{i}}{2}\left(\chi^{T} \partial_{t} \chi+\xi^{T} \partial_{t} \xi\right)-\mathrm{i} \bar{\theta}\left[\gamma^{8} \partial_{t}+Q_{1} \gamma^{1}+P_{1} \gamma^{2}+Q_{2} \gamma^{3}+P_{2} \gamma^{4}\right. \\
& \left.+Q_{3} \gamma^{5}+P_{3} \gamma^{6}+b \gamma^{9}-v t \gamma^{7}\right] \theta=-\frac{\mathrm{i}}{2}\left(\chi^{T} \partial_{t} \chi+\xi^{T} \partial_{t} \xi\right)-\mathrm{i} \bar{\theta} D_{\theta} \theta,
\end{aligned}
$$

where the 16 component spinors $\chi$ and $\xi$ (real) and $\theta$ (complex) describe the fermionic fluctuations of the Majorana-Weyl spinor $\psi$ arranged according to

$$
\delta \psi=\left(\begin{array}{cc}
\chi & \theta \\
\theta^{\dagger} & \xi
\end{array}\right)
$$

In Eq. (3.4) $\bar{\theta}=\theta^{\dagger} \gamma^{8}$ as follows from the specific representation of $\Gamma^{0}$ we have chosen. Just like in the open string formalism, the first contribution to the interaction between two D-branes is given by the one-loop vacuum energy $E$. In our case only the off-diagonal fluctuations of $\psi$ give a non-vanishing result, since the fields $\chi$ and $\xi$ are massless, and thus the whole calculation reduces to the evaluation of the functional determinant of $D_{\theta}$. It is easy to see from Eq. (3.4) that the lagrangian for the field $\theta$ is very similar to the one encountered in the computation of the static interaction between a D0 and a D8 brane. In fact, in this latter case, one chooses, for the bosonic degrees of freedom, a background with four pairs of operators satisfying the commutation relations (3.3); however, in the D0-D6 brane scattering, the velocity formally plays the same role of a gauge field $c_{i}$, since the background values for $X^{9}$ and $X^{0}$ are proportional to non-commuting operators of 
the same type of those appearing in the other directions. In order to make the analogy more evident, we perform a Wick rotation and introduce the euclidean "velocity" $\mathrm{v}=-\mathrm{i} v$, so that the $\theta$-part of the lagrangian becomes

$$
L_{\theta}=-\mathrm{i} \bar{\theta}\left[\mathrm{i} \gamma^{8} \partial_{\mathrm{t}}+Q_{1} \gamma^{1}+P_{1} \gamma^{2}+Q_{2} \gamma^{3}+P_{2} \gamma^{4}+Q_{3} \gamma^{5}+P_{3} \gamma^{6}+b \gamma^{9}-\mathrm{v} \mathrm{t} \gamma^{7}\right] \theta
$$

Now we can introduce a fourth pair of operators $Q_{4}=-\mathrm{vt}$ and $P_{4}=\mathrm{i} \partial_{\mathrm{t}}$ such that

$$
\left[Q_{4}, P_{4}\right]=\mathrm{iv}
$$

and thus the operator $D_{\theta}$ can be written as

$$
\begin{aligned}
D_{\theta} & =\left[b \gamma_{9}+m h\right], \\
m h & =\left[Q_{1} \gamma^{1}+P_{1} \gamma^{2}+Q_{2} \gamma^{3}+P_{2} \gamma^{4}+Q_{3} \gamma^{5}+P_{3} \gamma^{6}+Q_{4} \gamma^{7}+P_{4} \gamma^{8}\right] .
\end{aligned}
$$

This lagrangian was studied in detail in Refs. [15, 16] and here we will simply recall its main feature: from each eigenvector of $D_{\theta}\left(D_{\theta}|V\rangle_{r}=\lambda_{r}|V\rangle_{r}\right)$ that is not chiral $\left(\gamma^{9}|V\rangle \neq \pm|V\rangle\right)$ another eigenvector with opposite eigenvalue can be constructed; on the contrary $D_{\theta}$ has only one chiral eigenvector. In fact, it is easy to check that $|V\rangle_{-r}=\left(b-\lambda_{r} \gamma^{9}\right)|V\rangle_{r}$ is also an eigenvector of $D_{\theta}$ with eigenvalue $\left(-\lambda_{r}\right)$. However, this construction fails if $\gamma^{9}|V\rangle_{c}= \pm|V\rangle_{c}$ : in this case, $m$ must vanish when it acts on $|V\rangle_{c}$ (because it always changes the chirality of a vector) and $\lambda_{c}$ is simply equal to $\pm b$, thus, the combination $|V\rangle_{-c}$ vanishes. If, for the sake of simplicity, we suppose that the background values are positive $\left(c_{i}, \mathrm{v}>0\right)$, this unpaired eigenvector $|V\rangle_{c}$ is chiral $\gamma^{9}|V\rangle_{c}=|V\rangle_{c}$. The presence of the zero-mode of $m$ makes the evaluation of the determinant of $D_{\theta}$ a bit delicate. In fact, as it was pointed out in Ref. [16], when $b$ is zero the chiral eigenvector is a zero-mode for the full lagrangian and thus must be projected out in the definition of the determinant. Assuming that this projection has to be done also when $b$ is adiabatically switched on, we compute the effective energy as follows

$$
E \equiv \operatorname{Tr}^{\prime}\left[\ln \left(-\mathrm{i} D_{\theta}\right)\right]=\operatorname{Tr}\left[\left(\frac{1-\gamma^{9}}{2}\right) \ln \left(b^{2}+m h^{2}\right)\right]
$$

where the identity $b^{2}+m h^{2}=\left(\mathrm{i} D_{\theta}\right)\left(-\mathrm{i} D_{\theta}\right)$ and the pairing of the non chiral eigenvectors of $D_{\theta}$ have been used. Using the explicit expression of $m$ and the commutation relations of the operators $Q_{i}$ and $P_{i}$, we obtain

$$
E=\sum_{N_{1}, N_{2}, N_{3}, N_{4}= \pm 1} \operatorname{tr}\left[\left(\frac{1-N_{1} N_{2} N_{3} N_{4}}{2}\right) \log \left(b^{2}+\sum_{i=1}^{4} H_{i}-\sum_{i=1}^{3} c_{i} N_{i}-\mathrm{v} N_{4}\right)\right]
$$

where $N_{i}=-\mathrm{i} \gamma^{2 i-1} \gamma^{2 i}$, and $H_{i}=P_{i}^{2}+Q_{i}^{2}$. The sum over $N_{i}$ 's represents the trace over the 16 dimensional spinor space, and the symbol tr in Eq. (3.10) means the trace over the eigenstates of the harmonic oscillator hamiltonians $H_{i}$, whose 
spectrum is $2 c_{i}\left(n_{i}+1 / 2\right)$ for $i=1,2,3$ and $2 \mathrm{v}\left(n_{4}+1 / 2\right)$ for $i=4$. Then, using the "Schwinger" parametrization for the logarithm and summing over $n_{i}$, we get

$$
E=-\int_{0}^{\infty} \frac{d s}{s} \mathrm{e}^{-s b^{2}} \frac{1}{2}\left[\left(\prod_{i=1}^{3} \frac{\cosh c_{i} s}{\sinh c_{i} s}\right) \frac{\cosh \mathrm{v} s}{\sinh \mathrm{v} s}-1\right]
$$

By undoing the Wick rotation, the first term in the square bracket together with the contribution of the bosonic determinants computed for example in Ref. [10], correctly reproduces the even part of the interaction potential. On the contrary, the last term in the square bracket of Eq. (3.11), which is independent of the gauge fields $c_{i}$ of the D6 brane, arises because of the insertion of the chiral projector and accounts for the Lorentz-like magnetic interaction. By the usual trick

$$
1=v \sqrt{\frac{s}{\pi}} \int_{-\infty}^{\infty} \mathrm{e}^{-s(v \tau)^{2}} d \tau
$$

we can reintroduce the velocity dependence and verify that this term reproduces the same contribution that in string theory calculation came from the R-R odd spin structure. In fact,

$$
E_{\text {odd }}=\frac{v}{2 \sqrt{\pi}} \int_{-\infty}^{\infty} d \tau \int_{0}^{\infty} \frac{d s}{s^{1 / 2}} \mathrm{e}^{-s\left(b^{2}+(v \tau)^{2}\right)}=\frac{v}{2} \int_{-\infty}^{\infty} \frac{1}{r} d \tau
$$

leading to the same potential of Eqs. (1.7) and (2.24).

It is interesting to see what happens if one includes also the contribution of the chiral zero-mode of $m$, thus computing the full one-loop effective action $\Gamma$ of the matrix model. To expose the effect more clearly, it is convenient to consider a general background in which both $X_{7}$ and $X_{8}$ are constant and different from zero. In this case, the impact parameter becomes a vector $\vec{b}$ with components $b_{7}$ and $b_{8}$, and the operator $D_{\theta}$ in Eq. (3.8) gets replaced by $D_{\theta}=-\mathrm{i} b_{7} 11+b_{8} \gamma_{9}+m$. Using the properties we have mentioned above, it is not difficult to see that

$$
\begin{aligned}
\Gamma & \equiv \operatorname{Tr}\left[\ln \left(-\mathrm{i} D_{\theta}\right)\right]=\operatorname{Tr}^{\prime}\left[\ln \left(-\mathrm{i} D_{\theta}\right)\right]+\ln \left(b_{7}+\mathrm{i} b_{8}\right) \\
& =\frac{1}{2}\left(\operatorname{Tr}\left[\ln \left(\vec{b}^{2}+m h^{2}\right)\right]-\ln \vec{b}^{2}\right)+\ln \left(b_{7}+\mathrm{i} b_{8}\right) \\
& =-\int_{0}^{\infty} \frac{d s}{s} \mathrm{e}^{-s \vec{b}^{2}} \frac{1}{2}\left(\prod_{i=1}^{3} \frac{\cosh c_{i} s}{\sinh c_{i} s}\right) \frac{\cosh \mathrm{v} s}{\sinh \mathrm{v} s}+\mathrm{i} \operatorname{Im} \ln \left(b_{7}+\mathrm{i} b_{8}\right)
\end{aligned}
$$

The two terms of this expression represent the even and the odd parts of the effective action which account respectively for Coulomb-like and Lorentz-like interactions of the D0-D6 brane system. Notice that the last term in Eq. (3.14) exactly coincides with the expression in Eq. (1.6) obtained with the probe-source formalism, and correctly describes the phase-shift produced by a Lorentz-like interaction.

In conclusion we have shown both in string and in M(atrix) theory that the interaction between between pairs of Hodge dual D-branes in relative motions contains a term due to the Lorentz force. 


\section{Acknowledgements}

We thank M. Bertolini, I. Pesando and C. A. Scrucca for very useful discussions, and R. Iengo for useful remarks.

\section{References}

[1] J. Polchinski, Phys. Rev. Lett 75 (1995) 4724, hep-th/9510017; J. Polchinski, S. Chaudhuri and C.V. Johnson, Notes on D-branes, hep-th/ 9602052; J. Polchinski, TASI lectures on D-branes, hep-th/9611050.

[2] M. R. Douglas, D. Kabat, P. Pouliot, S. H. Shenker, Nucl. Phys. B485 (1997) 85 , hep-th/9608024.

[3] T. Banks, W. Fischler, S. H. Shenker, L. Susskind, Phys. Rev. D55 (1997) 5112, hep-th/9610043.

[4] A. Bilal, M(atrix) Theory : a Pedagogical Introduction, hep-th/9710136; D. Bigatti and L. Susskind, Review of Matrix Theory, hep-th/9712072; W. Taylor, Lectures on D-branes, gauge theory and M(atrices), hep-th/ 9801182.

[5] M. Billó, P. Di Vecchia, M. Frau, A. Lerda, I. Pesando, R. Russo and S. Sciuto, Microscopic string analysis of the D0-D8 brane system and dual $R-R$ states, hep-th/9802088.

[6] M. Billó, D. Cangemi and P. Di Vecchia, Phys. Lett. 400B (1997) 63, hep-th/9701190.

[7] M. Frau, I. Pesando, S. Sciuto, A. Lerda and R. Russo, Phys. Lett. 400B (1997) 52, hep-th/9702037.

[8] P. Di Vecchia, M. Frau, I. Pesando, S. Sciuto, A. Lerda, R. Russo, Nucl. Phys. B507 (1997) 259, hep-th/9707068.

[9] C. Bachas, Phys. Lett. 374B (1996) 37, hep-th/9511043. G. Lifschytz, Phys. Lett. 388B (1996) 720, hep-th/9604156

[10] G. Lifschytz, Four brane and six brane interactions in M(atrix) theory, hep-th/9612223.

[11] J. M. Pierre, Phys.Rev. D56 (1997) 6710, hep-th/9707102.

[12] V. Balasubramanian, F. Larsen, Nucl. Phys. B506 (1997) 61 hep-th/9703039.

[13] E. Keski-Vakkuri, P. Kraus, Nucl. Phys. B510 (1997) 199, hep-th/9706196. 
[14] M. Bertolini, R. Iengo and C. A. Scrucca, Electric and magnetic interaction of dyonic D-branes and odd spin structure, hep-th/9801110.

[15] P. M. Ho, M. Li and Y. S. Wu, $p-p^{\prime}$ strings in M(atrix) theory, hep-th/ 9706073 .

[16] P. M. Ho and Y. S. Wu, Brane creation in M(atrix) theory, hep-th/9708137.

[17] I. Chepelev, A. A. Tseytlin, Long-distance interactions of branes: correspondence between supergravity and super Yang-Mills descriptions, hep-th/ 9709087 .

[18] J. F. Morales, C. A. Scrucca and M. Serone, Phys. Lett. 417B (1998) 233, hep-th/9709063; Scale independent spin-effects in D-brane dynamics, hep-th/9801183.

[19] C. P. Bachas, M. R. Douglas and M. B. Green, JHEP 07 (1997) 002, hep-th/ 9705074; U. Danielsson, G. Ferretti and I. R. Klebanov, Phys. Rev. Lett. 79 (1997) 1984, hep-th/ 9705084; O. Bergman, M. Gaberdiel and G. Lifschytz, Nucl. Phys. B509 (1998) 194, hep-th/9705130. 